\title{
Reduction of Midline Shift Following Decompressive Hemicraniectomy for Malignant Middle Cerebral Artery Infarction
}

\author{
Sang-Beom Jeon, ${ }^{\mathrm{a}}$ Sun U. Kwon, ${ }^{\mathrm{a}}$ Jung Cheol Park, ${ }^{\mathrm{b}}$ Deok Hee Lee, ${ }^{\mathrm{c}}$ Sung-Cheol Yun, ${ }^{\mathrm{d}}$ Yeon-Jung Kim, ${ }^{\mathrm{a}}$ \\ Jae-Sung Ahn, ${ }^{\mathrm{b}}$ Byung-Duk Kwun, ${ }^{\mathrm{b}}$ Dong-Wha Kang, ${ }^{\mathrm{a}} \mathrm{H}$. Alex Choi, ${ }^{\mathrm{e}}$ Kiwon Lee, ${ }^{\mathrm{e}}$ Jong S. Kim ${ }^{\mathrm{a}}$ \\ aDepartment of Neurology, Asan Medical Center, University of Ulsan College of Medicine, Seoul, Korea \\ ${ }^{b}$ Department of Neurosurgery, Asan Medical Center, University of Ulsan College of Medicine, Seoul, Korea \\ 'Department of Radiology, Asan Medical Center, University of Ulsan College of Medicine, Seoul, Korea \\ dDivision of Epidemiology and Biostatistics Clinical Research Center, Asan Medical Center, University of Ulsan College of Medicine, Seoul, Korea \\ 'Departments of Neurology and Neurosurgery, TheUniversity of Texas Medical School at Houston, Houston, Texas, USA
}

\begin{abstract}
Background and Purpose Hemicraniectomy is a decompressive surgery used to remove a large bone flap to allow edematous brain tissue to bulge extracranially. However, early indicators of the decompressive effects of hemicraniectomy are unclear. We investigated whether reduction of midline shift following hemicraniectomy is associated with improved consciousness and survival in patients with malignant middle cerebral artery infarctions.

Methods We studied 70 patients with malignant middle cerebral artery infarctions (MMI) who underwent hemicraniectomies. Midline shift was measured preoperatively and postoperatively using computed tomography (CT). Consciousness level was evaluated using the Glasgow Coma Scale on postoperative day 1. Patient survival was assessed six months after stroke onset.

Results The median time interval between preoperative and postoperative CT was 8.3 hours (interquartile range, 6.1-10.2 hours). Reduction in midline shift was associated with higher postoperative Glasgow Coma Scale scores $(P<0.05)$. Forty-three patients $(61.4 \%)$ were alive at six months after the stroke. Patients with reductions in midline shifts following hemicraniectomy were more likely to be alive at six months post-stroke than those without $(P<0.001)$. Reduction of midline shift was associated with lower mortality at six months after stroke, after adjusting for age, sex, National Institutes of Health Stroke Scale score, and preoperative midline shift (adjusted hazard ratio, 0.71; 95\% confidence interval, 0.62-0.81; $P<0.001$ ).

Conclusions Reduction in midline shift following hemicraniectomy was associated with improved consciousness and six-month survival in patients with MMI. Hence, it may be an early indicator of effective decompression following hemicraniectomy.
\end{abstract}

Keywords Middle cerebral artery; Infarction; Decompressive craniectomy; Mortality

\section{Introduction}

Malignant middle cerebral artery (MCA) infarction (MMI) is a large hemispheric infarction due to the occlusion of the proximal

\author{
Correspondence: Sang-Beom Jeon \\ Department of Neurology, Asan \\ Medical Center, University of Ulsan \\ College of Medicine, 88 Olympic-ro \\ 43-gil, Songpa-gu, Seoul 05505, Korea \\ Tel: +82-2-3010-3440 \\ Fax: +82-2-474-4691 \\ E-mail: sbjeonmd@gmail.com
}

Received: April 8, 2016 Revised: June 4, 2016 Accepted: June 30, 2016

The authors have no financial conflicts of interest.

\footnotetext{
Copyright (ㅇ 2016 Korean Stroke Society

This is an Open Access article distributed under the terms of the Creative Commons Attribution Non-Commercial License (http://creativecommons.org/licenses/by-nc/4.0/) which permits unrestricted non-commercial use, distribution, and reproduction in any medium, provided the original work is properly cited.
} 
on the side of the MMI and allows the infarcted brain tissue to bulge extracranially. Recent clinical trials have clearly demonstrated benefits of hemicraniectomy in increasing survival rates. $^{6-10}$ However, $20-40 \%$ of patients still die following hemicraniectomy due to refractory brain swelling. ${ }^{2,6-10}$

MMI patients indicated for surgical decompression typically have decreased levels of consciousness. ${ }^{2-4,6-10}$ Space-occupying cerebral edema due to MMI can displace the infarcted hemisphere to the opposite side, resulting in an altered mental status. Brain edema as manifested by lateral displacement of the brain, i.e., midline shift, is an important cause of the decrease in consciousness and poor clinical outcomes in patients with MMI. ${ }^{11}$ However, it is unknown whether reduction in midline shift by hemicraniectomy can improve consciousness and survival in patients with MMI.

Herein, we aimed to investigate whether reduction of midline shift following hemicraniectomy is associated with improved consciousness and survival in patients with MMI.

\section{Methods}

\section{Patients}

We considered stroke patients admitted to the neurological intensive care unit of Asan Medical Center from January 2005 to August 2014. Patients were included in this study if 1) they were diagnosed as having ischemic stroke involving $>66 \%$ of the MCA territory, as assessed by a computed tomography (CT) scan, and 2) if hemicraniectomy was performed. Patients with additional infarctions in the anterior and/or posterior cerebral artery territories were not excluded, but those with parenchymal hematomas ( $\mathrm{PH} 1$ and $\mathrm{PH} 2$ ) on preoperative $\mathrm{CT}$ scan were excluded to rule out the effects of the hematoma and its evacuation on the midline shift. ${ }^{12}$ This study was approved by the institutional review board of Asan Medical Center. The need for written informed consent was waived because of the retrospective nature of this study.

\section{Clinical assessments}

We obtained data on demographics, risk factors, laboratory findings, the National Institutes of Health Stroke Scale (NIHSS), and Glasgow Coma Scale (GCS) from our stroke registry and medical records. ${ }^{13,14}$ The onset of stroke was considered to be the time when a patient was last known to be without ischemic symptoms. When a patient's symptoms were mild and the infarct size was initially small but progressed to a large hemispheric infarction, the onset of stroke was still considered to be the lastknown time that the patient was normal. Stroke subtypes were determined according to the classification of the Trial of Org
10172 in Acute Stroke Treatment. ${ }^{15}$ Patient survival outcomes were assessed on post-stroke day 180 . Survival status and the date of death were obtained from the National Health Insurance Corporation.

\section{Surgical and medical management}

All patients with MMI were admitted to the neurological intensive care unit. The decision to perform hemicraniectomy was based on our protocol and discussions among neurologists, neurosurgeons, and the patients' family members (Supplementary Figure 1). The surgery consisted of a large hemicraniectomy and a duroplasty at the side of the MMI. A large bony window was created by removing a bone flap in the frontal, parietal, temporal, and occipital regions (Supplementary Figure 2). The dura was opened and fixed at the margin of the craniotomy to prevent epidural bleeding. An expansive duroplasty was then performed using an artificial dura mater. Non-contrast CT was routinely performed after the surgery before or immediately after transfer to the neurological intensive care unit. Medical management of patients with $\mathrm{MMI}$ at our hospital has previously been detailed. ${ }^{5}$ Perioperative medical treatments included general management, such as mechanical ventilation for patients with abnormal respiratory drives or reflexes, head elevation to $30^{\circ}$, avoidance of hypoxia and hyponatremia, and control of fever and hyperglycemia; sedation and analgesia as indicated; hyperventilation before urgent decompressive hemicraniectomy; osmotherapy with mannitol or hypertonic saline; and induced hypothermia and barbiturate coma therapy for patients with refractory brain swelling despite decompressive hemicraniectomy. All patients underwent neurological examinations, including GCS determination, every hour during their stay in the intensive care unit.

\section{Imaging analysis}

Non-contrast CT scans were routinely obtained on admission, immediately before hemicraniectomy, immediately after hemicraniectomy, upon neurological deterioration, at discharge from the intensive care unit, and at the discretion of the attending physician. For our study purposes, we used the findings of the last preoperative CT and the first postoperative CT. The slice thickness of the axial CT was $5 \mathrm{~mm}$. Midline shift was determined using the distance from the midline to the septum pellucidum (midline shift at the level of the septum pellucidum, SPS) and to the pineal gland (midline shift at the level of the pineal gland, PGS). ${ }^{11,16}$ The reduction in midline shift (SPS and PGS) in millimeters was defined as the amount $(\mathrm{mm})$ of midline shift on the last preoperative CT minus that of the first postoperative CT. We used midline shift in millimeters for our analysis of midline shift, but we also categorized changes in midline shift as reduc- 
tions (reduction of $\geq 1 \mathrm{~mm}$ ), no change $(-1 \mathrm{~mm}<$ reduction $<1$ $\mathrm{mm}$ ), and progression (reduction of $\leq-1 \mathrm{~mm}$ ) for further analysis. Skull size was measured using the lateral view of the skull radiograph. The maximal size of the bone flap was measured between opposing hemicraniectomy edges on serial axial slices of post-hemicraniectomy CTs. The bone flap size in this study was typically smaller than the maximal diameter of the same bone flap measured intraoperatively, ${ }_{1}^{4}$ as we did not measure the distance on the surface of the skull intra-operatively or using the skull radiograph, but measured this distance on CT post-operatively (Supplementary Figure 3). The extracranial bulging volume was measured as the volume of the brain tissue beyond the bony window, and the extracranial bulging ratio was defined as the ratio of the extracranial bulging volume to the maximal size of the bone flap (Figure 1). The presence and maximal thickness of subgaleal hematomas were also evaluated.

Brain magnetic resonance imaging (MRI) was routinely performed at the time of the initial diagnosis of stroke. The detailed MRI protocol has been previously published..$^{17}$ The infarct volume was measured using diffusion-weighted imaging (DWI) as the sum of the infarct area in each slice multiplied by the slice thickness.

We used a built-in calculation tool in the picture archiving and communication system (PACS; PetaVision ${ }^{\mathrm{TM}}$, Seoul, Korea) of the hospital for measurements of CT and MRI findings. CT and $M R I$ variables were measured by investigators blind to our clinical data and patient outcomes. Imaging findings were measured by two independent investigators (S.-B..J. and Y.-J.K.) and agreement was determined using intraclass correlation coefficients.

\section{Data analysis}

We compared baseline characteristics and imaging variables according to the survival status of the patients at 180 days poststroke. We used the Pearson $\chi^{2}$ test or Fisher's exact test for categorical variables, Student's $t$-test or Mann-Whitney $U$ test for continuous variables, Pearson coefficient for calculating correlations, and the Jonckheere-Terpstra test for ordered variables, as appropriate. Variables with a $P$ value $<0.1$ by univariate analysis
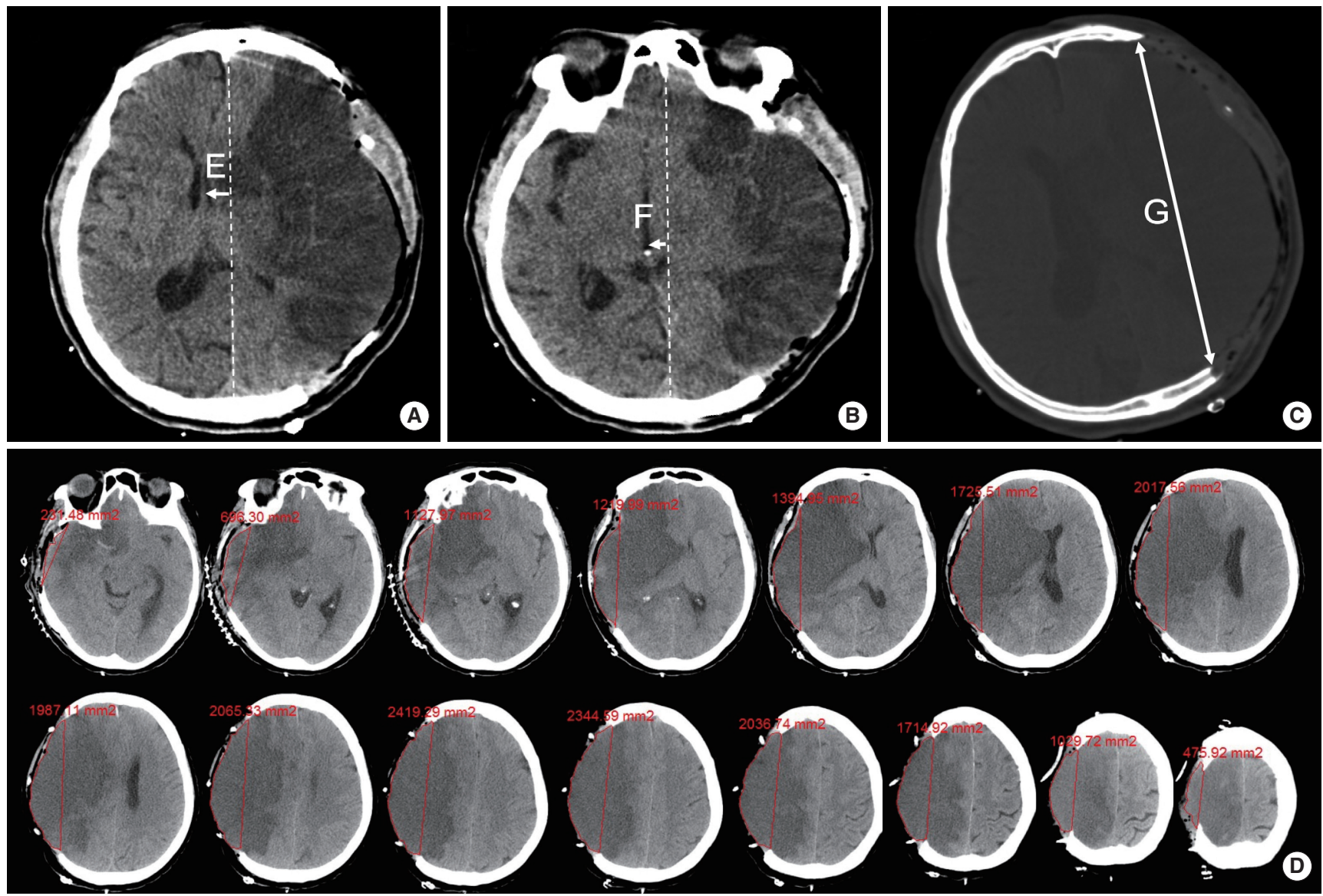

Figure 1. Postoperative CT measurements. Septum pellucidum shift (SPS, "E") was determined using the distance from the midline to the septum pellucidum at the level of the fornix (A). Pineal gland shift (PGS, "F") was determined using the distance from the midline to the pineal gland (B). Maximal size of the bone flap (" $\mathrm{G}$ ") was measured between opposing hemicraniectomy edges on serial axial slices on the post-hemicraniectomy CT (C). The extracranial bulging volume was measured as the volume of the brain tissue beyond the bony window (areas marked with red lines) and the extracranial bulging ratio was defined as the ratio of the extracranial bulging volume to the maximal size of the bone flap (D). 
were included in a multivariate model as candidate variables and then removed using a backward stepwise selection procedure. Preoperative (SPS and PGS) and postoperative (reduction of SPS and PGS) CT findings were also included in the multivariate models. Survival curves were constructed using Kaplan-Meier estimates and were compared using the log-rank test. Clinical and imaging variables were evaluated using Cox proportional hazard analysis to identify predictors of survival. Hazard ratios (HRs) with 95\% confidence interval ( $\mathrm{Cls}$ ) were also calculated. The discrimination ability and the contributions of SPS and PGS to the prediction of survival at 180 days post-stroke were evaluated using the c-index. Statistical significance was defined as a two-tailed $P$ value of $<0.05$. All statistical analyses were performed using SPSS version 21 (IBM Corp., Armonk, NY, USA) and SAS ${ }^{\circledast}$ version 9.1 (SAS Institute Inc., Cary, NC, USA).

\section{Results}

During the study period, 2,518 patients with ischemic stroke were admitted to our neurological intensive care unit. Of these, 75 underwent hemicraniectomy for MMI. Five patients with parenchymal hematomas as seen on preoperative CT were excluded. Thus, 70 patients were included in the final analysis.

\section{Baseline characteristics}

Of the 70 patients included in the study, 37 (52.9\%) were men. The median patient age was 63 years (range, 22-81 years; interquartile range [IQR], 52.8-69.0 years). On admission, the median NIHSS was $15.5(I Q R, 12.8-19.0)$ and the GCS total score was 10.0 (IQR, 8.0-13.0). At the time of surgery, the median GCS total score was 8.0 (IQR, 7.0-11.0). The median time from the last-known normal time to the skin incision under anesthesia was 41.6 hours (IQR, 26.2-59.3 hours). The median time from the last preoperative $\mathrm{CT}$ to the first postoperative $\mathrm{CT}$ was 8.3 hours (IQR, 6.1-10.2 hours). At 180 days post-stroke, 43 (61.4\%) of the patients were alive and 27 (38.6\%) were deceased. Baseline characteristics, including demographics, risk factors, stroke classifications, vital signs, and laboratory findings, were not different between the surviving and the deceased patients, except that deceased patients were more likely to be females, have higher NIHSS scores, and have lower GCS scores (Table 1).

\section{Surgical and medical treatments}

Hemicraniectomy with duroplasty was performed in 70 study patients. There were no statistically significant factors related to survival among the preoperative medical treatments, including thrombolysis, use of antithrombotic agents and statins, endotra-

Table 1. Patient characteristics and factors associated with 6-month mortality

\begin{tabular}{|c|c|c|c|c|c|}
\hline \multirow{2}{*}{ Variables } & \multirow{2}{*}{ Total $(n=70)$} & \multicolumn{2}{|c|}{ Mortality at 6 months } & \multirow{2}{*}{$\mathrm{HR}(95 \% \mathrm{Cl})$} & \multirow{2}{*}{$P$} \\
\hline & & No $(n=43)$ & Yes $(n=27)$ & & \\
\hline \multicolumn{6}{|l|}{ Demographics and risk factors } \\
\hline Age (year) & $63.0(52.8-69.0)$ & $63.0(51.0-69.0)$ & $65.0(54.0-69.0)$ & $1.01(0.98-1.04)$ & 0.489 \\
\hline Sex, male & $37(52.9)$ & $28(65.1)$ & $9(33.3)$ & $0.36(0.16-0.81)$ & 0.013 \\
\hline Hypertension & $39(55.7)$ & $26(60.5)$ & $13(48.1)$ & $0.70(0.33-1.49)$ & 0.353 \\
\hline Diabetes mellitus & $11(15.7)$ & $6(14.0)$ & $5(18.5)$ & $1.35(0.51-3.56)$ & 0.548 \\
\hline Hypercholesterolemia & $21(30.0)$ & $11(25.6)$ & $10(37.0)$ & $1.64(0.75-3.58)$ & 0.217 \\
\hline Smoking & $20(28.6)$ & $14(32.6)$ & $6(22.2)$ & $0.59(0.24-1.47)$ & 0.261 \\
\hline Previous stroke & $10(14.3)$ & $5(11.6)$ & $5(18.5)$ & $1.51(0.57-4.00)$ & 0.405 \\
\hline \multicolumn{6}{|l|}{ Stroke classification and neurologic status } \\
\hline Cardioembolism & $48(68.6)$ & $30(69.8)$ & $17(63.0)$ & $0.83(0.37-1.85)$ & 0.648 \\
\hline NIHSS score on admission & $15.5(12.8-19.0)$ & $15.0(12.0-18.0)$ & $18.0(13.0-20.0)$ & $1.09(1.01-1.17)$ & 0.027 \\
\hline GCS score before craniectomy & $8.0(7.0-11.0)$ & $9.0(7.0-12.0)$ & $7.0(6.0-11.0)$ & $0.86(0.74-0.99)$ & 0.030 \\
\hline \multicolumn{6}{|l|}{ Initial imaging findings } \\
\hline Stroke in dominant hemisphere & $27(38.6)$ & $16(37.2)$ & $11(40.7)$ & $1.13(0.52-2.43)$ & 0.760 \\
\hline Infarction limited to the MCA territory & $28(40.0)$ & $19(44.2)$ & $9(33.3)$ & $0.74(0.33-1.65)$ & 0.462 \\
\hline Complete occlusion of the MCA $(n=65)$ & $48(73.8)$ & $28(65.1)$ & $20(74.1)$ & $0.99(0.42-2.35)$ & 0.986 \\
\hline Infarct volume on DWI, $\mathrm{cm}^{3}(\mathrm{n}=65)$ & $246.0(206.5-313.5)$ & $226.0(193.0-286.5)$ & $261.5(215.5-333.5)$ & $1.01(1.00-1.01)$ & 0.016 \\
\hline \multicolumn{6}{|l|}{ Perioperative CT findings } \\
\hline Preoperative SPS (mm) & $9.5(5.2-13.5)$ & $11.0(6.0-13.5)$ & $7.1(4.7-11.5)$ & $0.95(0.88-1.03)$ & 0.235 \\
\hline Preoperative PGS (mm) & $3.6(1.3-5.9)$ & $3.8(1.7-6.2)$ & $3.0(1.1-5.8)$ & $1.00(0.87-1.15)$ & 0.970 \\
\hline Reduction in SPS (mm) & $0.7(-1.0-3.5)$ & $2.6(0.1-5.1)$ & $-0.4(-4.8-0.6)$ & $0.79(0.72-0.87)$ & $<0.001$ \\
\hline Reduction in PGS (mm) & $0.4(-0.3-1.4)$ & $1.0(0-2.6)$ & $-0.2(-1.4-0.3)$ & $0.76(0.66-0.87)$ & $<0.001$ \\
\hline
\end{tabular}

Data are given as a number (column percent), median value (interquartile range), or ratio (95\% confidence interval).

HR, hazard ratio; Cl, confidence interval; NIHSS, National Institutes of Health Stroke Scale; GCS, Glasgow Coma Scale; MCA, middle cerebral artery; DWI, diffusion-weighted imaging; CT, computed tomography; SPS, septum pellucidum shift; PGS, pineal gland shift. 
cheal intubation, mechanical ventilation, and osmotherapy. Factors associated with the timing of the surgery (workdays vs. holidays and daytime vs. nighttime) and the operators were not related to survival.

\section{Imaging findings and survival}

Before surgery, brain MRI was performed in 65 (92.9\%) patients, magnetic resonance angiography or CT angiography was performed in 65 patients, and non-contrast CT was performed in 70 patients. After surgery, non-contrast CT was obtained for all 70 patients.

Findings of the initial MRI, the last preoperative $\mathrm{CT}$, and the first postoperative CT are presented in Table 1. Large infarct vol- umes as seen by DWI were associated with mortality at 180 days post-stroke. Reductions in midline shift (SPS and PGS) were significantly associated with higher levels of consciousness as measured by GCS at 24 hours post-operation and were marginally associated with an improvement of consciousness as measured by postoperative GCS minus preoperative GCS (Figure 2, Supplementary Figure 4). Patients with reductions in midline shift (both in millimeter units as measured and in categorized groups) were more likely to survive at 180 days post-stroke than those with progression or no changes in midline shift $(P<0.001)$ (Table 1 , Figure 3). Twenty-nine out of the 34 patients (85.3\%) with a reduction in SPS, 10 out of the 19 patients (52.6\%) with no change in SPS, and 4 out of the 17 patients (23.5\%) with a pro-
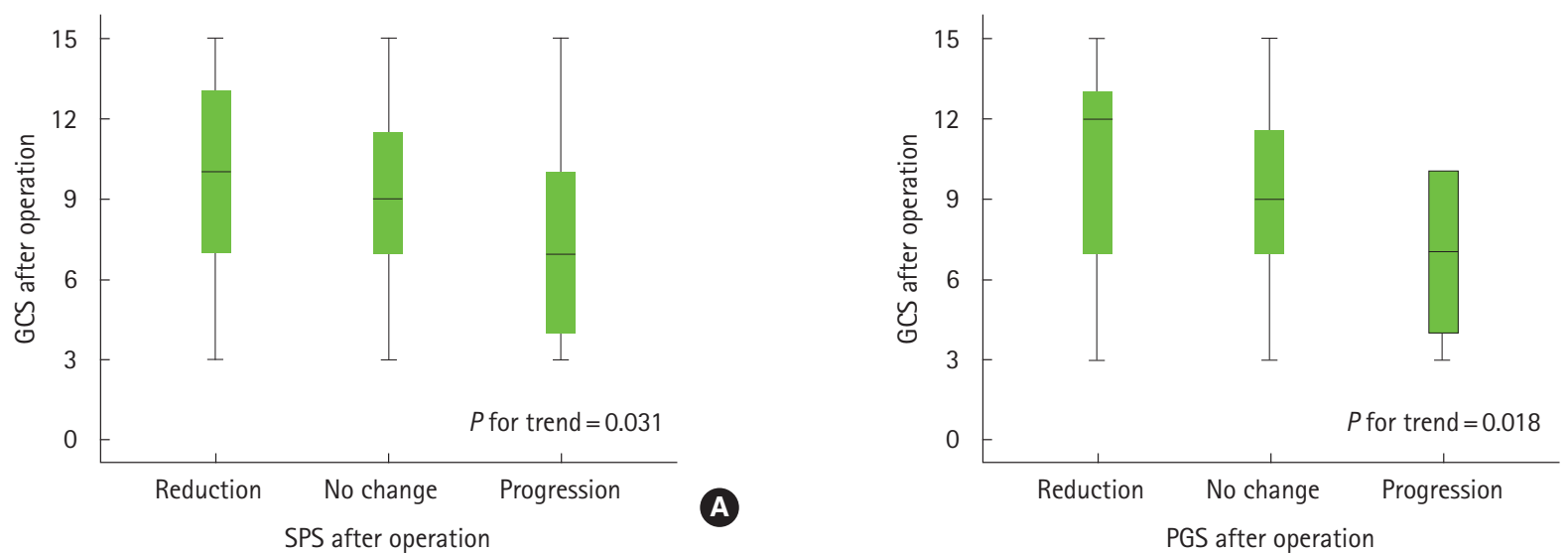

Figure 2. Reduction in midline shift and level of consciousness after hemicraniectomy. The Glasgow Coma Scale (GCS) is shown in the box plots according to the reduction in midline shift at the septum pellucidum (SPS, A) and the pineal gland (PGS, B) following hemicraniectomy. GCS scores were measured 24 hours after the hemicraniectomy.
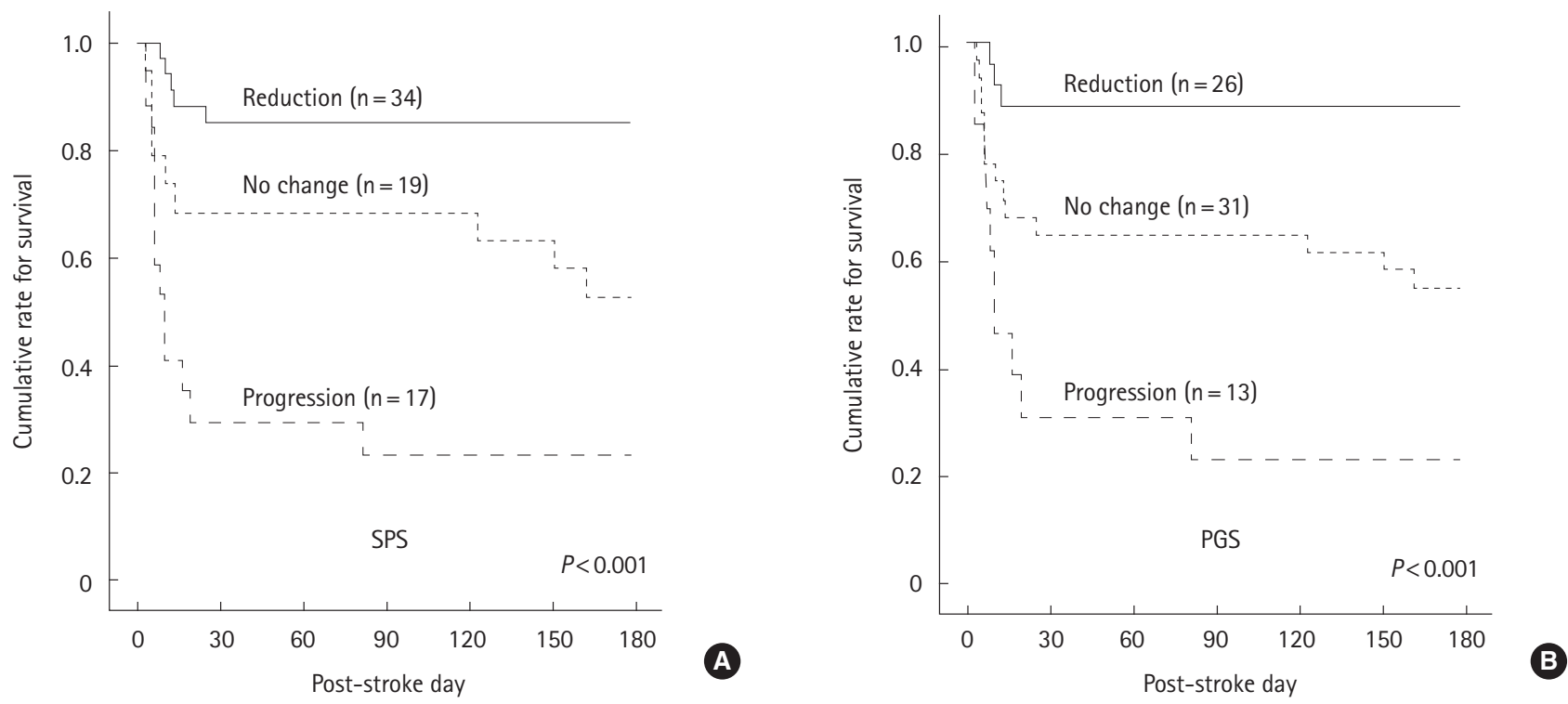

Figure 3. Reduction in midline shift and 6-month survival outcomes. Kaplan-Meier curves showing the proportions of surviving patients stratified by the change in midline shift at the septum pellucidum (SPS, A) and at the pineal gland (PGS, B) after hemicraniectomy. The curves differed significantly according to the log-rank test. 


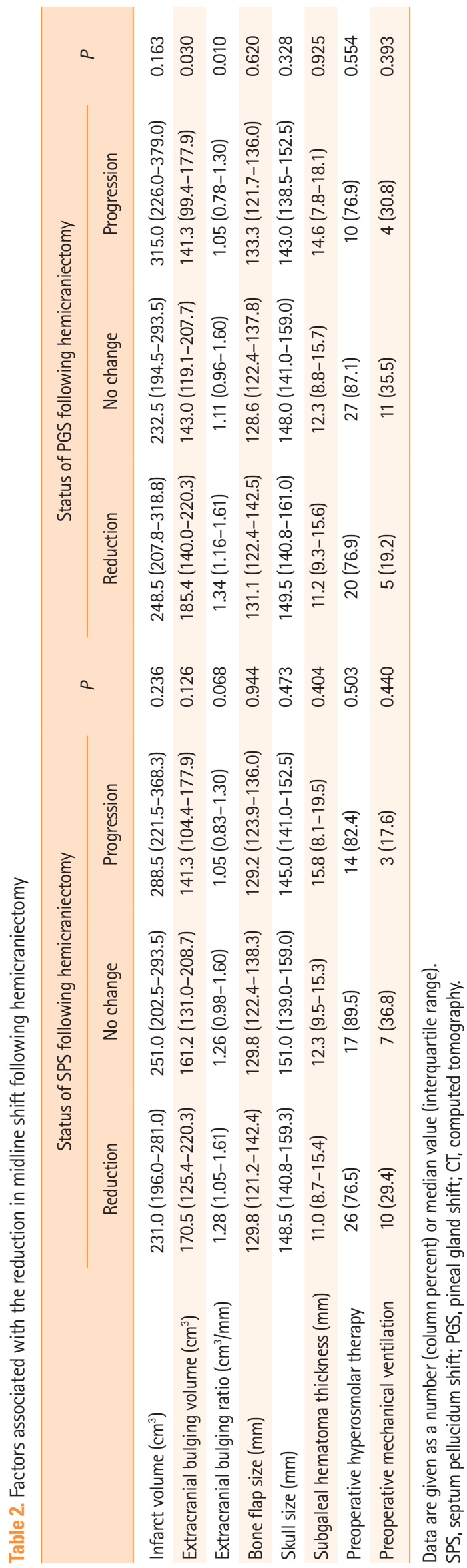

gression of SPS were alive at 180 days post-stroke. Twenty-three out of the 26 patients (88.5\%) with reductions in PGS, 17 out of the 31 patients (54.8\%) with no change in PGS, and 3 out of the 13 patients (23.1\%) with progression of PGS at the first postoperative CT were alive 180 days post-stroke. The intraclass correlation coefficient between the two $\mathrm{CT}$ raters was high (0.985 for SPS, $P<0.001 ; 0.991$ for PGS, $P<0.001$; and 0.993 for extracranial bulging, $P<0.001)$.

We also analyzed radiological factors related to the reduction in the midline shift. Extracranial bulging volume was significantly related to the reduction in PGS (categorical variable, $P=0.030$; continuous variable, $P=0.021$ ) and marginally related to the reduction in SPS (categorical variable, $P=0.126$; continuous variable, $P=0.022$ ). The extracranial bulging ratio was also significantly related to the reduction in PGS (categorical variable, $P=0.010$; continuous variable, $P=0.007$ ) and marginally related to the reduction in SPS (categorical variable, $P=0.068$; continuous variable, $P=0.011$ ). However, infarct volume on $D W I$, skull size, bone flap size, subgaleal hematoma thickness, time interval

Table 3. Factors associated with 6-month mortality on multivariable analysis

\begin{tabular}{|c|c|c|c|}
\hline & \multicolumn{2}{|c|}{ 6-month mortality } & \multirow{2}{*}{$\begin{array}{l}\text { C-index } \\
(95 \% \mathrm{Cl})\end{array}$} \\
\hline & $\begin{array}{l}\text { Adjusted HR } \\
(95 \% \mathrm{Cl})\end{array}$ & $P$ & \\
\hline Model 1 & & & $0.849(0.793-0.904)$ \\
\hline Sex (male) & $0.45(0.20-1.02)$ & 0.055 & \\
\hline $\begin{array}{l}\text { NIHSS score on } \\
\text { admission }\end{array}$ & $1.12(1.04-1.22)$ & 0.005 & \\
\hline Preoperative SPS (mm) & $1.17(1.05-1.30)$ & 0.005 & \\
\hline Reduction in SPS (mm) & 0.71 & $<0.001$ & \\
\hline Model 2 & & & $0.805(0.733-0.877)$ \\
\hline Sex (male) & $0.43(0.18-1.03)$ & 0.058 & \\
\hline $\begin{array}{l}\text { NIHSS score on } \\
\text { admission }\end{array}$ & $1.09(1.01-1.19)$ & 0.031 & \\
\hline Preoperative PGS (mm) & $1.16(1.00-1.34)$ & 0.051 & \\
\hline Reduction in PGS (mm) & 0.76 & $<0.001$ & \\
\hline Model 3 & & & $0.846(0.790-0.902)$ \\
\hline Age & $1.01(0.97-1.05)$ & 0.760 & \\
\hline Sex (male) & $0.47(0.20-1.13)$ & 0.091 & \\
\hline $\begin{array}{l}\text { NIHSS score on } \\
\text { admission }\end{array}$ & $1.13(1.04-1.22)$ & 0.005 & \\
\hline Preoperative SPS (mm) & $1.17(1.05-1.30)$ & 0.005 & \\
\hline Reduction in SPS (mm) & $0.71(0.62-0.80)$ & $<0.001$ & \\
\hline Model 4 & & & $0.808(0.737-0.878)$ \\
\hline Age & $1.01(0.96-1.05)$ & 0.826 & \\
\hline Sex (male) & $0.46(0.17-1.27)$ & 0.132 & \\
\hline $\begin{array}{l}\text { NIHSS score on } \\
\text { admission }\end{array}$ & $1.09(1.01-1.19)$ & 0.032 & \\
\hline Preoperative PGS (mm) & $1.16(1.00-1.34)$ & 0.050 & \\
\hline Reduction in PGS (mm) & $0.76(0.65-0.89)$ & 0.001 & \\
\hline
\end{tabular}

$\mathrm{HR}$, hazard ratio; $\mathrm{Cl}$, confidence interval; NIHSS, National Institutes of Health Stroke Scale; SPS, septum pellucidum shift; PGS, pineal gland shift. C-index for base model (sex and NIHSS) $=0.690(0.603-0.777)$. 
from preoperative to postoperative $\mathrm{CT}$, preoperative use of hyperosmotic agents, and preoperative mechanical ventilation were not related to changes in SPS and PGS (Table 2).

After adjustment for sex, NIHSS, and preoperative SPS, reduction in SPS was associated with lower mortality 180 days poststroke in the Cox proportional hazards model (adjusted HR, 0.71; $95 \% \mathrm{Cl}, 0.62-0.81 ; P<0.001)$. Reduction in PGS was also associated with lower mortality at 180 days post-stroke after adjustments for sex, NIHSS, and preoperative PGS (adjusted HR, 0.76; $95 \% \mathrm{Cl}, 0.66-0.89 ; P<0.001)$. We further analyzed these data with adjustments for age and sex, NIHSS, and preoperative midline shift, and found similar results (Table 3, Supplementary Table 1).

\section{Discussion}

We analyzed the association between a reduction in midline shift following hemicraniectomy and the level of consciousness and six-month survival in 70 patients with MMl. The reduction of midline shift was evaluated a median of 0.4 hours after the operation and a median of 8.3 hours after preoperative CT. Since such time intervals are short, we believe that the reduction in midline shift was driven mostly by the surgical decompression. A reduction in midline shift was associated with higher levels of postoperative consciousness. A reduction in midline shift was further related to survival: approximately $85 \%$ of the study patients with a reduced midline shift, 50\% of patients with a stationary midline shift, and $20 \%$ of patients with a progressed midline shift survived at six months. Thus, a reduction in midline shift may be a good indicator of the decompression effect of the hemicraniectomy and may be an early predictor of long-term survival in patients with $\mathrm{MMI}$.

Midline shift has been correlated with the level of consciousness in patients with acute brain lesions. An early study of midline shifts reported a strong association between it and the development of stupor and coma in patients with acute intracerebral hemorrhages, subdural hematomas, epidural hematomas, and large MCA infarctions." A recent study showed that a reversal of midline shift was related to awakening from coma in patients whose cause of brain edema was heterogeneous. ${ }^{18}$ Previous studies of midline shift have primarily focused on the development of and recovery from unconsciousness during the acute stage of brain edema in heterogeneous populations using various treatment modalities. ${ }^{11,18}$ In the present study, the midline shift was caused by brain edema following acute ischemic stroke, and its reduction was an immediate effect of the surgical decompression.

Several radiological characteristics have been proposed as predictors of mortality in patients with MCA infarctions, including large MCA infarct volumes, the involvement of multiple vascular territories, occlusion of the internal cerebral artery, hyperdense MCA signs on CT, M1 susceptibility vessel signs on gradient-echo imaging, large perfusion defects, and the absence of underlying brain atrophy. ${ }^{19-23}$ Such radiological characteristics predict a malignant course in patients who receive medical treatments. However, predictors of mortality after hemicraniectomy have not been clearly determined, although substantial proportions of patients still die of refractory brain swelling after hemicraniectomy. Early predictors of survival may have clinical implications in triggering additional timely interventions. ${ }^{3-5}$ Given that refractory brain swelling is the main cause of death in patients with $\mathrm{MMI}$, it is expected that patients who still expire despite hemicraniectomy would finally develop a prominent midline shift. ${ }^{23,24}$ In the present study, however, a reduction in midline shift was evaluated within one hour after a hemicraniectomy. Thus, the reduction in midline shift was an immediate effect of the hemicraniectomy. To our knowledge, our study is the first to show that an immediate reduction of midline shift following a hemicraniectomy may be an early predictor of survival in MMI patients.

Systematic studies to evaluate factors associated with reductions in midline shift are lacking. In the present analysis, extracranial bulging was significantly related to the reduction of PGS and marginally related to the reduction of SPS. Unarguably, producing a large bony window by the removal of the bone flap may result in extracranial bulging of swollen brain tissue. The reduction in midline shift following hemicraniectomy may result from changing the direction of the brain displacement from the intracranial side to the extracranial side. However, massive extracranial bulging may be the result of severe brain swelling as well. Thus, the efficacy of surgical decompression should be interpreted using the reduction in midline shift and not solely based on extracranial bulging. Radiological and clinical implications of extracranial bulging are undetermined at present. Future prospective studies are needed to address this issue.

In addition to the inherent limitations of a retrospective design and selection bias, our study has other limitations of note. First, patient outcomes were defined as long-term survival and not as functional outcomes, which were not available for this retrospective study. However, the primary purpose of a hemicraniectomy is to save the life of a stroke victim. We do not believe that the mortality rate in our patients was driven by a self-fulfilling prophecy, as withdrawal of care was not allowed for local legal and cultural reasons. Second, detailed operative factors were not obtained due to the retrospective nature of our analyses. The shape and size of the duroplasty, three-dimensional information regarding the bone flap such as surface area and the maximal length of the bone flap in the surgical field, resection of the tem- 
poralis muscle, and strokectomy were not analyzed. However, these factors eventually affect the reduction in midline shift. Third, we estimated the infarct volume based on initial DWI. Most of our subjects had large DWI volumes, in agreement with previous studies..$^{25}$ The outcomes of a hemicraniectomy are more likely to be influenced by the last preoperative infarct volume than by the initial infarct volume. However, the final preoperative infarct volume contributes to the preoperative midline shift. Thus, we believe that additional data on final infarct volume would not have led to different findings. Fourth, the mortality rate of our patients may have been affected by the retrospective design and selection bias. We included patients whose infarct size was initially small but progressed to a large hemispheric infarction. Such patients were not likely included in previous clinical trials. Moreover, the patients in the earlier study period were enrolled before an early hemicraniectomy strategy, which was based on clinical trials and guidelines, was employed. Fifth, assessment of the level of consciousness may have been affected by the use of sedative agents during the perioperative period.

\section{Conclusions}

We showed that a reduction in midline shift following hemicraniectomy was associated with improved consciousness and survival in patients with MMl. Our findings suggest that reductions in midline shift may be early indicators of effective decompression following hemicraniectomy. Further studies are necessary to validate our findings and to explore their clinical implications with regard to triggering additional and timely interventions after a hemicraniectomy.

\section{References}

1. Hacke W, Schwab S, Horn M, Spranger M, De Georgia M, von Kummer R. 'Malignant' middle cerebral artery territory infarction: clinical course and prognostic signs. Arch Neurol 1996; 53:309-315.

2. Wartenberg KE. Malignant middle cerebral artery infarction. Curr Opin Crit Care 2012;18:152-163.

3. Wijdicks EF, Sheth KN, Carter BS, Greer DM, Kasner SE, Kimberly WT, et al. American Heart Association Stroke Council. Recommendations for the management of cerebral and cerebellar infarction with swelling: a statement for healthcare professionals from the American Heart Association/American Stroke Association. Stroke 2014;45:1222-1238.

4. Torbey MT, Bösel J, Rhoney DH, Rincon F, Staykov D, Amar AP, et al. Evidence-based guidelines for the management of large hemispheric infarction: a statement for health care profes- sionals from the neurocritical care society and the german society for neuro-intensive care and emergency medicine. Neurocrit Care 2015;22:146-164.

5. Jeon SB, Koh Y, Choi HA, Lee K. Critical care for patients with massive ischemic stroke. J Stroke 2014;16:146-160.

6. Vahedi K, Vicaut E, Mateo J, Kurtz A, Orabi M, Guichard J, et al. Sequential-design, multicenter, randomized, controlled trial of early decompressive craniectomy in malignant middle cerebral artery infarction (DECIMAL Trial). Stroke 2007;38:25062517.

7. Jüttler $E$, Schwab $S$, Schmiedek $P$, Unterberg $A$, Hennerici $M$, Woitzik J, et al. Decompressive Surgery for the Treatment of Malignant Infarction of the Middle Cerebral Artery (DESTINY): a randomized, controlled trial. Stroke 2007;38:2518-2525.

8. Vahedi K, Hofmeijer J, Juettler E, Vicaut E, George B, Algra A, et al. Early decompressive surgery in malignant infarction of the middle cerebral artery: a pooled analysis of three randomised controlled trials. Lancet Neurol 2007;6:215-222.

9. Hofmeijer J, Kappelle $\sqcup$, Algra A, Amelink GJ, van Gijn J, van der Worp HB, et al. Surgical decompression for space-occupying cerebral infarction (the Hemicraniectomy After Middle Cerebral Artery infarction with Life-threatening Edema Trial [HAMLET]): a multicentre, open, randomised trial. Lancet Neurol 2009;8:326-333.

10. Jüttler $E$, Unterberg $A$, Woitzik J, Bösel J, Amiri H, Sakowitz $\mathrm{OW}$, et al. Hemicraniectomy in older patients with extensive middle-cerebral-artery stroke. N Engl J Med 2014;370:10911100.

11. Ropper AH. Lateral displacement of the brain and level of consciousness in patients with an acute hemispheral mass. N Engl J Med 1986;314:953-958.

12. Fiorelli M, Bastianello $S$, von Kummer R, del Zoppo GJ, Larrue $V_{1}$ Lesaffre $E_{1}$ et al. Hemorrhagic transformation within 36 hours of a cerebral infarct: relationships with early clinical deterioration and 3-month outcome in the European Cooperative Acute Stroke Study I (ECASS I) cohort. Stroke 1999;30: 2280-2284.

13. Brott T, Adams HP, Jr., Olinger CP, Marler JR, Barsan WG, Biller $J$, et al. Measurements of acute cerebral infarction: a clinical examination scale. Stroke 1989;20:864-870.

14. Teasdale $G$, Jennett B. Assessment and prognosis of coma after head injury. Acta Neurochir (Wien) 1976;34:45-55.

15. Adams HP Jr, Bendixen BH, Kappelle ப, Biller J, Love BB, Gordon $D L$, et al. Classification of subtype of acute ischemic stroke. Definitions for use in a multicenter clinical trial. TOAST. Trial of Org 10172 in Acute Stroke Treatment. Stroke 1993;24: 35-41.

16. Quattrocchi KB, Prasad P, Willits NH, Wagner FC Jr. Quantifi- 
cation of midline shift as a predictor of poor outcome following head injury. Surg Neurol 1991;35:183-188.

17. Jeon SB, Kang DW, Kim JS, Kwon SU. Homocysteine, smallvessel disease, and atherosclerosis: an MRI study of 825 stroke patients. Neurology 2014;83:695-701.

18. Kowalski RG, Buitrago MM, Duckworth J, Chonka ZD, Puttgen $H A$, Stevens RD, et al. Neuroanatomical predictors of awakening in acutely comatose patients. Ann Neurol 2015;77:804816.

19. Shimoyama T, Kimura K, Uemura J, Yamashita S, Saji N, Shiba-

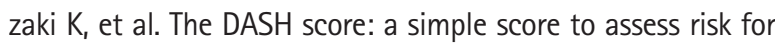
development of malignant middle cerebral artery infarction. $J$ Neurol Sci 2014;338:102-106.

20. Dohmen C, Galldiks N, Bosche B, Kracht L, Graf R. The severity of ischemia determines and predicts malignant brain edema in patients with large middle cerebral artery infarction. Cerebrovasc Dis 2012;33:1-7.

21. Manno EM, Nichols DA, Fulgham JR, Wijdicks EF. Computed tomographic determinants of neurologic deterioration in patients with large middle cerebral artery infarctions. Mayo Clin
Proc 2003;78:156-160.

22. Beck C, Kruetzelmann A, Forkert ND, Juettler E, Singer OC, Köhrmann $M$, et al. A simple brain atrophy measure improves the prediction of malignant middle cerebral artery infarction by acute DWI lesion volume. J Neurol 2014;261:1097-1103.

23. Jaramillo A, Gongora-Rivera F, Labreuche J, Hauw JJ, Amarenco P. Predictors for malignant middle cerebral artery infarctions: a postmortem analysis. Neurology 2006;66:815-820.

24. Tu PH, Liu ZH, Chuang CC, Yang TC, Wu CT, Lee ST. Postoperative midline shift as secondary screening for the long-term outcomes of surgical decompression of malignant middle cerebral artery infarcts. J Clin Neurosci 2012;19:661-664.

25. Tu PH, Liu ZH, Chuang CC, Yang TC, Wu CT, Lee ST. Postoperative midline shift as secondary screening for the long-term outcomes of surgical decompression of malignant middle cerebral artery infarcts. J Clin Neurosci 2012;19:661-664.

25. Oppenheim C, Samson Y, Manaï R, Lalam T, Vandamme $X$, Crozier $S$, et al. Prediction of malignant middle cerebral artery infarction by diffusion-weighted imaging. Stroke 2000;31:21752181. 


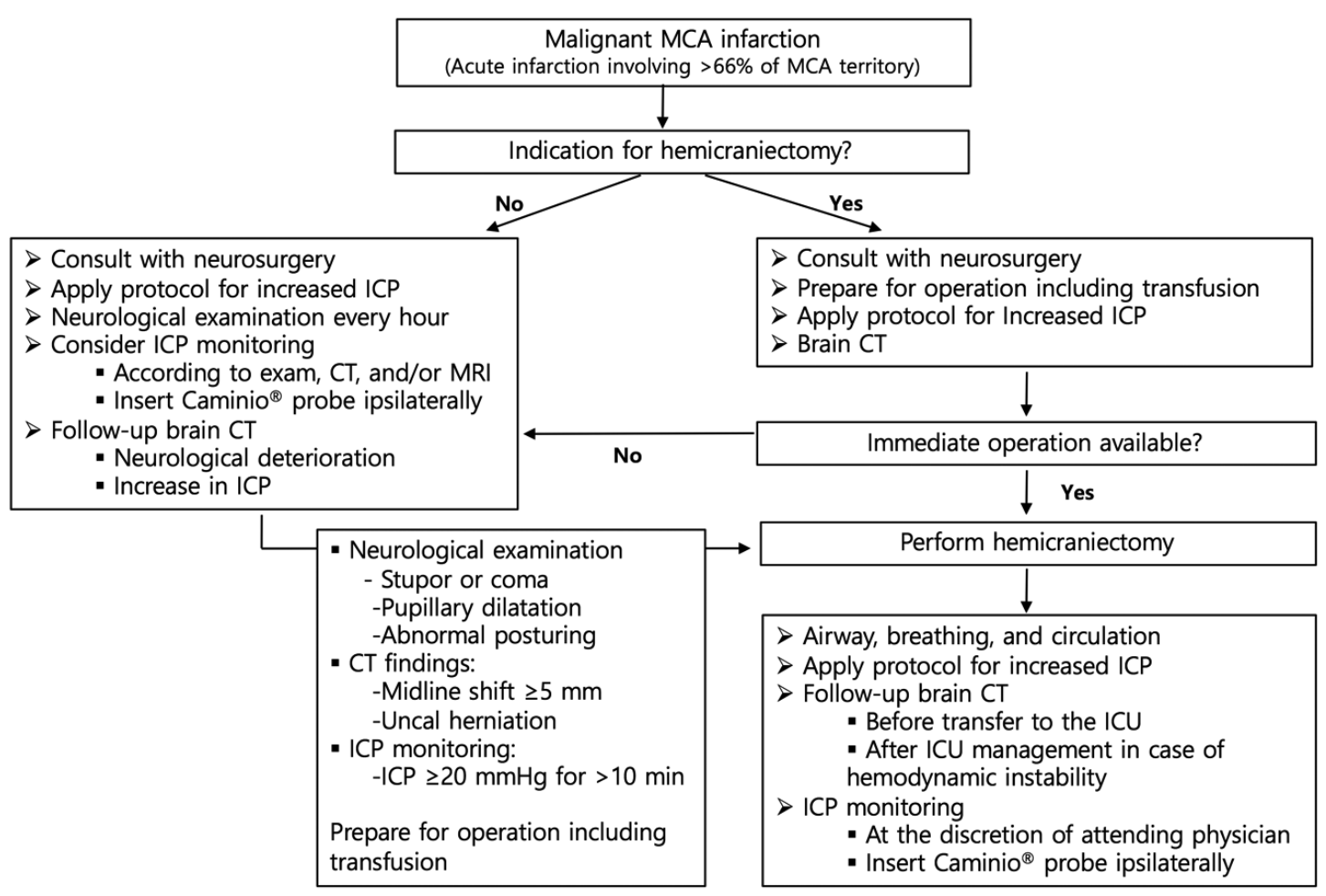

Supplementary Figure 1. Management algorithm for malignant middle cerebral artery infarction. MCA indicates middle cerebral artery; ICP, intracranial pressure; ICU, intensive care unit; CT, computed tomography. 


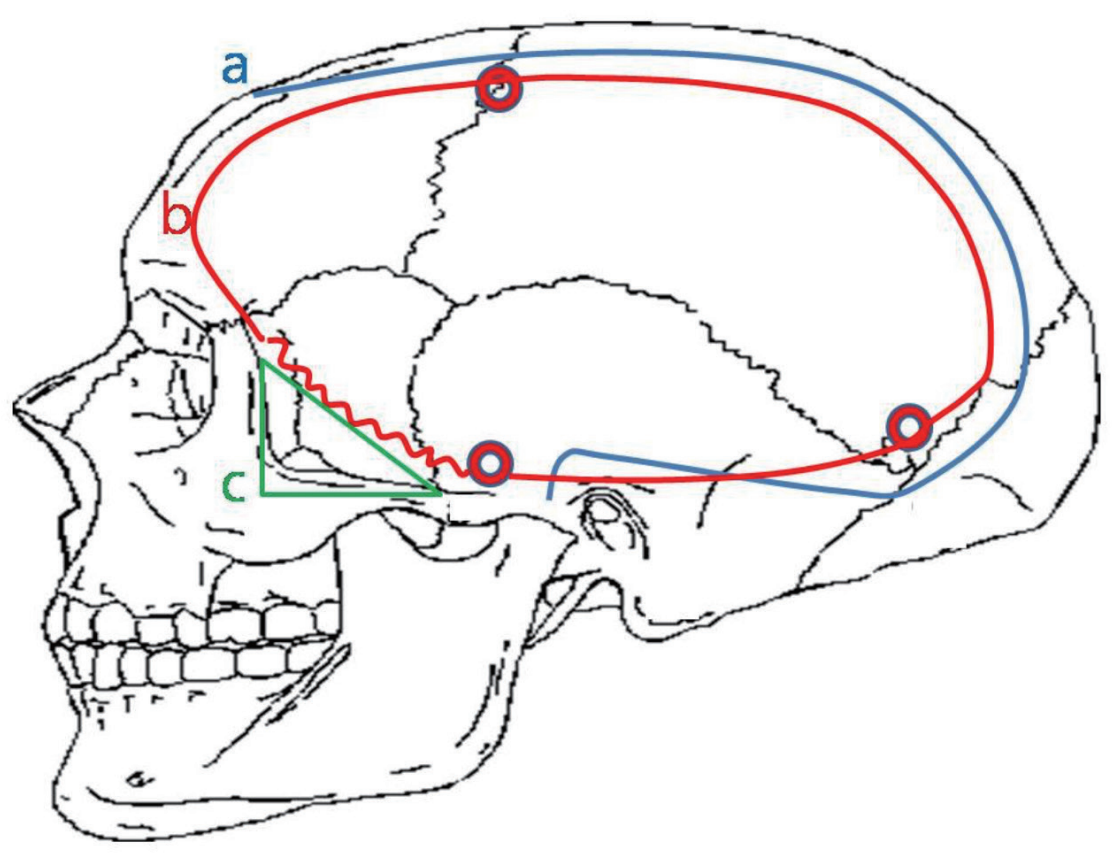

Supplementary Figure 2. Incision lines for the hemicraniectomy. The blue line shows a question-mark-shaped skin incision line (a). The skin, temporalis muscle, fascia, and periosteum are retracted after the incision. The red line indicates the margin for skull incision (b). Three red circles demonstrate points for major burr holes. A large bony window was created by removing a bone flap in the frontal, parietal, temporal, and occipital regions. The green line is for the additional resection of parts of the temporal bone (c). The dura was opened and fixed at the margin of the craniotomy to prevent epidural bleeding. An expansive duroplasty was then carried out using an artificial dura mater. Resection of the temporalis muscle and the infarcted brain tissue was not routinely performed. 


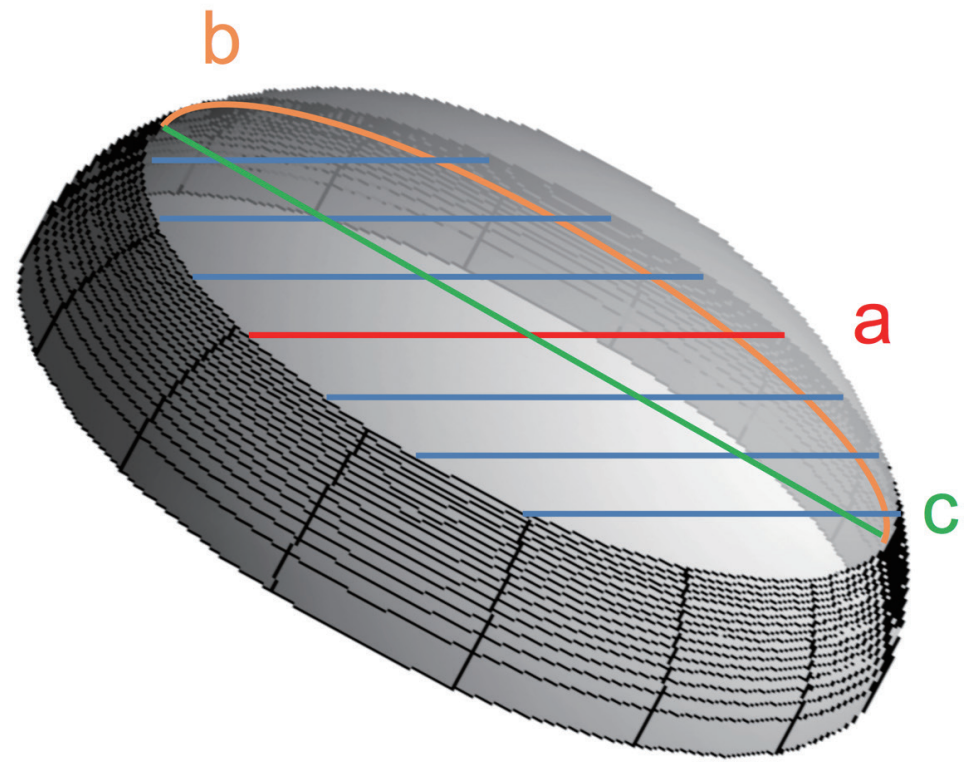

Supplementary Figure 3. Maximal length of the bone flap. The size of bone flap was determined using the longest distance between opposing hemicraniectomy edges on axial cuts of postoperative CT for a bone flap with an oval shape. Blue parallel lines indicate lines connecting two opposing edges on serial axial cuts of post-hemicraniectomy CT, and the largest blue lines is marked in red. We used a red line (a) to indicate a bone flap size that is typically shorter than that measured intraoperatively (b) or on a skull radiograph (c). 

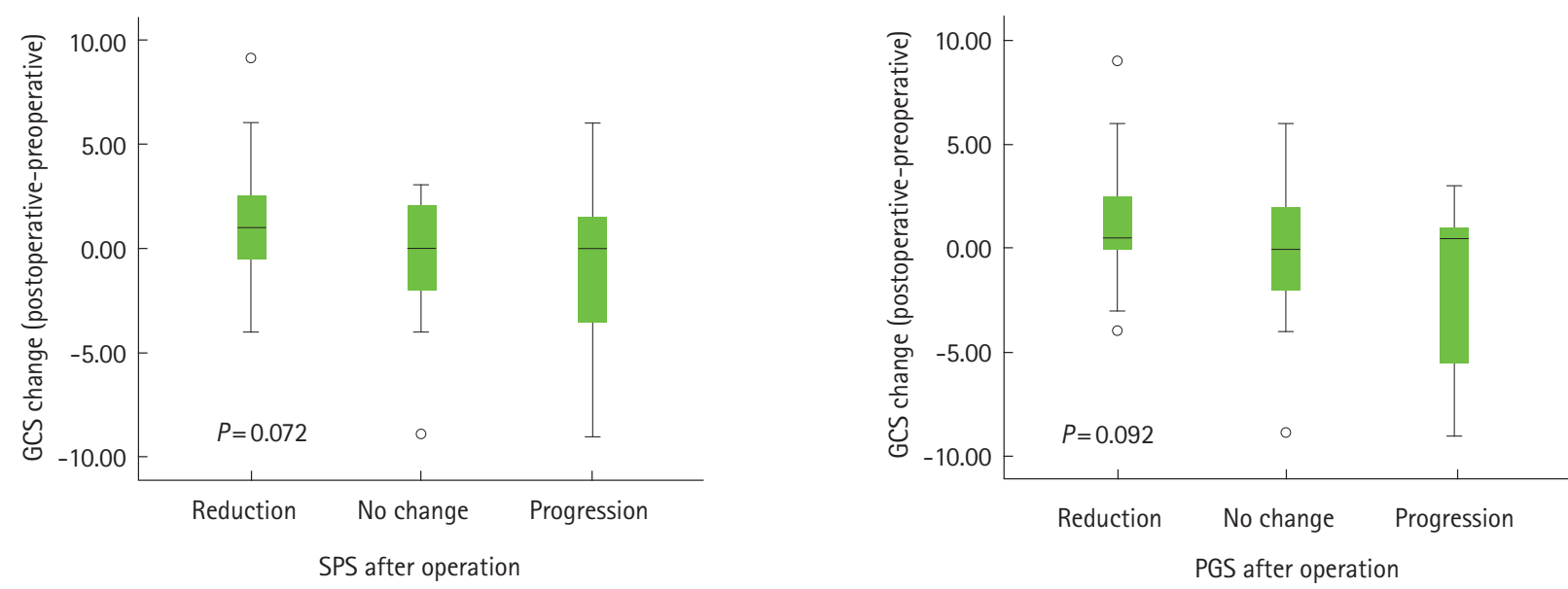

Supplementary Figure 4. Reduction in midline shift and improvement of consciousness after hemicraniectomy. GCS, Glasgow Coma Scale; SPS, midline shift (septum pellucidum shift); PGS, midline shift (pineal gland shift). 
Supplementary Table 1. Factors associated with mortality at six months using multiple logistic regression analyses

\begin{tabular}{|c|c|c|}
\hline & \multicolumn{2}{|c|}{ Mortality at 180 days post-stroke } \\
\hline & Adjusted odds ratio (95\% Cl) & $P$ \\
\hline \multicolumn{3}{|l|}{ Model 1} \\
\hline Sex (male) & $0.25(0.06-1.00)$ & 0.050 \\
\hline NIHSS score on admission & $1.22(1.04-1.43)$ & 0.013 \\
\hline Preoperative SPS (mm) & $1.26(1.04-1.53)$ & 0.019 \\
\hline Reduction in SPS (mm) & $0.52(0.37-0.73)$ & $<0.001$ \\
\hline \multicolumn{3}{|l|}{ Model 2} \\
\hline Sex (male) & $0.30(0.09-0.99)$ & 0.048 \\
\hline NIHSS score on admission & $1.16(1.02-1.31)$ & 0.027 \\
\hline Preoperative PGS (mm) & $1.18(0.94-1.47)$ & 0.156 \\
\hline Reduction in PGS (mm) & $0.57(0.94-1.47)$ & 0.002 \\
\hline \multicolumn{3}{|l|}{ Model 3} \\
\hline Age & $1.03(0.95-1.11)$ & 0.509 \\
\hline Sex (male) & $0.31(0.07-1.46)$ & 0.138 \\
\hline NIHSS score on admission & $1.22(1.04-1.42)$ & 0.014 \\
\hline Preoperative SPS (mm) & $1.27(1.04-1.54)$ & 0.018 \\
\hline Reduction in SPS (mm) & $0.51(0.35-0.72)$ & $<0.001$ \\
\hline \multicolumn{3}{|l|}{ Model 4} \\
\hline Age & $1.01(0.96-1.07)$ & 0.645 \\
\hline Sex (male) & $0.34(0.09-1.25)$ & 0.103 \\
\hline NIHSS score on admission & $1.15(1.02-1.31)$ & 0.028 \\
\hline Preoperative PGS (mm) & $1.19(0.95-1.49)$ & 0.141 \\
\hline Reduction in PGS (mm) & $0.56(0.39-0.81)$ & 0.002 \\
\hline
\end{tabular}

$\mathrm{Cl}$, confidence interval; NIHSS, National Institutes of Health Stroke Scale; SPS, midline shift (septum pellucidum shift); PGS, midline shift (pineal gland shift). 